\title{
Realization of low-loss mirrors with sub-nanometer flatness for future gravitational wave detectors
}

\author{
C. Michel, N. Morgado, L. Pinard, B. Sassolas, R. Bonnand, J. Degallaix, D. Forest, R. Flaminio \\ Laboratoire des Matériaux Avancés- CNRS- \\ Bâtiment VIRGO- 7, avenue Pierre de Coubertin. 69622 VILLEURBANNE Cedex- France \\ G. Billingsley \\ LIGO - California Institute of Technology, Pasadena, CA 91125, USA \\ Corresponding author: c.michel@lma.in2p3.fr
}

\begin{abstract}
The second generation of gravitational wave detectors will aim at improving by an order of magnitude their sensitivity versus the present ones (LIGO and VIRGO). These detectors are based on long-baseline Michelson interferometer with high finesse Fabry-Perot cavity in the arms and have strong requirements on the mirrors quality. These large low-loss mirrors (340 $\mathrm{mm}$ in diameter, $200 \mathrm{~mm}$ thick) must have a near perfect flatness. The coating process shall not add surface figure Zernike terms higher than second order with amplitude $>0.5 \mathrm{~nm}$ over the central $160 \mathrm{~mm}$ diameter. The limits for absorption and scattering losses are respectively 0.5 and $5 \mathrm{ppm}$. For each cavity the maximum loss budget due to the surface figure error should be smaller than $50 \mathrm{ppm}$. Moreover the transmission matching between the two inputs mirrors must be better than $99 \%$.

We describe the different configurations that were explored in order to respect all these requirements. Coatings are done using IBS.

The two first configurations based on a single rotation motion combined or not with uniformity masks allow to obtain coating thickness uniformity around $0.2 \% \mathrm{rms}$ on $160 \mathrm{~mm}$ diameter. But this is not sufficient to meet all the specifications.

A planetary motion completed by masking technique has been studied. With simulated values the loss cavity is below 20 ppm, better than the requirements. First experimental results obtained with the planetary system will be presented.
\end{abstract}

Keyword: Ion beam sputtering, Large Low-losses optics, uniformity, planetary

\section{Introduction}

Ion beam sputtering (IBS) is well known as the unique technology able to reduce optical losses at ppm level [1]. Low losses optics with very high reflection value ( $\mathrm{R}>99.999 \%)$ were obtained by multilayer dielectric coating based on a stack of alternate tantala $\left(\mathrm{Ta}_{2} \mathrm{O}_{5}\right)$ layers and silica layers. A large coating chamber $(2.2 *$ $2.2 * 2 \mathrm{~m}^{3}$ ) (Fig 1) using IBS have been built by the Laboratoire des Matériaux Avancés (LMA) to coat large low-loss optics (350 mm diameter, $100 \mathrm{~mm}$ thick, $20 \mathrm{~kg}$ ) for the VIRGO gravitational wave detector [2]. This detector [3] operating at wavelength of $1064 \mathrm{~nm}$ is based on long-baseline Michelson interferometer with high finesse Fabry-Perot cavity in the arms. The most stringent specifications for the high reflective optics of the Fabry-Perot cavity (called Input Test Mass and End Test Mass) were the optical losses. The requirements have been finally exceeded with absorptions measured at $1 \mathrm{ppm}$ at $1064 \mathrm{~nm}$ and scattering losses around $5 \mathrm{ppm}$ [4]. These optics have been installed in the detector which has been exploited for several years up to 2011. Even if the sensitivity of the detector has reached the scheduled value [5] the accumulated data allowed only fixing upper limit for several gravitational wave sources, not to discover them. So a second generation of detectors called Advanced VIRGO [6] and Advanced LIGO [7] is under construction to improve the sensitivity by an order of magnitude. 


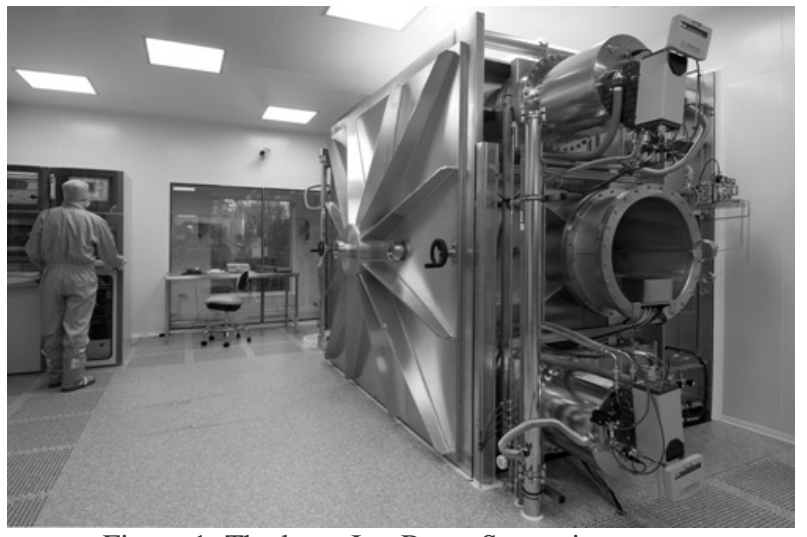

Figure 1: The large Ion Beam Sputtering coater

Limitations of Advanced VIRGO sensitivity come from different origins with frequency dependence (Fig 2). At low frequency the suspension thermal noise is the largest contribution for the limitation. Between 50 and $300 \mathrm{~Hz}$ the thermal noise induced by the coating is dominant. Tantalum pentoxide $\left(\mathrm{Ta}_{2} \mathrm{O}_{5}\right)$ traditionally used as high index material in the multilayer stack is the major contributor to the coating brownian noise [8]. To reach the requirements $\mathrm{Ta}_{2} \mathrm{O}_{5}$ will be replaced by Titanium doped Tantalum pentoxide $\left(\mathrm{Ti}^{\mathrm{T}} \mathrm{Ta}_{2} \mathrm{O}_{5}\right)$ with mechanical losses reduce by a factor 2 . Moreover the stack will be optimized to reduce the amount of the high index material [9]. At high frequency the quantum noise is dominant. To gain a factor 10 in sensitivity the solution is to introduce more power in the Fabry-Perot cavities (125 W instead of $17 \mathrm{~W}$ for VIRGO+) and increase the arm cavity Finesse (443 vs 150 in VIRGO +) but these changes increase the radiation pressure at low frequency. The use of heavier optics (40 kg vs 20 $\mathrm{kg}$ ) will help to limiting this effect.

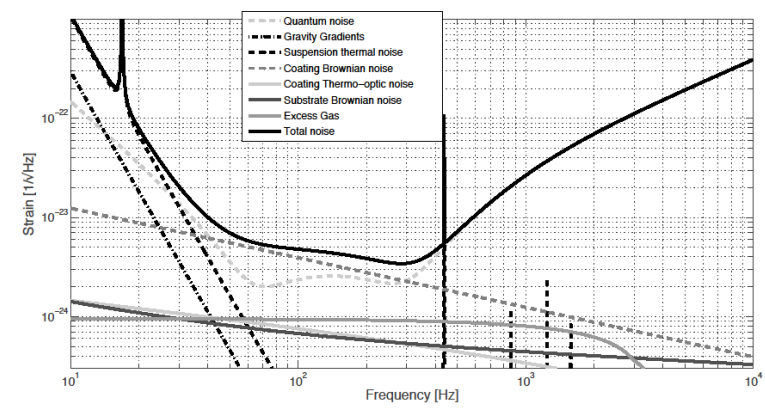

Figure 2: Advanced VIRGO sensitivity

The benefit of higher cavity finesses is limited by the round-trip optical losses (RTL) in the arm. So the specifications have been set to $75 \mathrm{ppm}$ for these losses which are due to the absorption in the multilayer coatings, the surface micro-roughness, the transmission of the end mirror and the small angle scattering. With $25 \mathrm{ppm}$ budgeted for the three first sources of losses, the RTL due to the surface figure error should be smaller than $50 \mathrm{ppm}$. Simulations have set $0.5 \mathrm{~nm} \mathrm{rms}$ for the flatness of the mirror on the central area $\varnothing 160$ $\mathrm{mm}$. Finally on the central $160 \mathrm{~mm}$ diameter the coating process shall not change the substrate sagitta by more than $8 \mathrm{~nm}$ and not add surface figure Zernike terms higher than second order with amplitude $>0.5 \mathrm{~nm}$. The limits for absorption and scattering losses are respectively 0.5 and $5 \mathrm{ppm}$. Moreover, in order to minimize the interferometer contrast defect, the transmission matching between the two input mirrors must be better than $1 \%: 2(\mathrm{~T} 1-\mathrm{T} 2) /(\mathrm{T} 1+\mathrm{T} 2)<0.01$ $\%$ with $0.013 \%<\mathrm{T}<0.015 \%)$.

In this paper we will describe the different techniques which have been tested in order to coat the new generation of optics with sub-nanometer flatness for gravitational wave detectors. The two first configurations were based on a single rotation motion for the substrate combined or not with an uniformity mask while the third configuration uses a planetary motion system.

\section{The twin mirrors:}

The first approach was an up-grade of the solution used for the coating of the optics of VIRGO+ [10]. In this solution two optics were coated simultaneously in the same batch in our large IBS coater chamber. The optics were positioned on a diameter around the axis of rotation of the substrate holder. Uniformity was obtained by traditional masking technique: a mask was placed between the deposition target and the rotating substrate. Due to the difference of the shape of the sputtered particles (the plume) it was necessary to use two masks: one for the silica layers and a second for the titanium doped tantalum pentoxide layers. We started the study with the masks used for VIRGO+. Because of the substrate thickness increase $(200 \mathrm{~mm}$ instead of 100 $\mathrm{mm}$ ) the substrate holder was modified to keep the same distance between the targets, the masks and the coated surface. With the glass slides used previously for VIRGO+ the sensitivity of the thickness measurement is limited at $0.3 \%$. For this new uniformity study we used silica substrates ( $25.4 \mathrm{~mm}$ diameter, $6 \mathrm{~mm}$ thick) positioned in the coated plan on fake metallic substrates. The samples were aligned along diameter of the fake substrate (Fig. 3). 


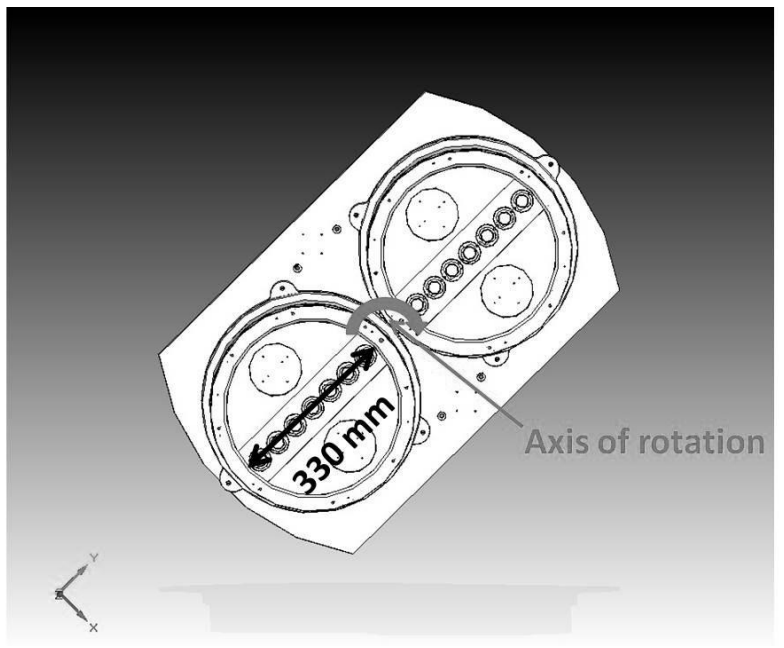

Fig 3: Twin mirrors configuration

The coating thickness measurement method is based on spectrophotometric analysis. Transmission was measured at the center of each sample with a spectrophotometer (Lambda 1050- Perkin Elmer) between 400 and $1400 \mathrm{~nm}$. The thickness was estimated by fitting the transmission curve with dedicated software like TFCalc [11] or Optical Constant [12]. For $\mathrm{Ti}: \mathrm{Ta}_{2} \mathrm{O}_{5}$ the analysis was made on a $500 \mathrm{~nm}$ thick layer. For the silica layers it was not possible to study directly a single layer deposited on silica substrates as there is no index contrast then no variation in transmission versus the wavelength. We obtain a large variation in transmission with a doublet composed of 56 $\mathrm{nm}$ of $\mathrm{Ti}: \mathrm{Ta}_{2} \mathrm{O}_{5}$ plus $720 \mathrm{~nm}$ of silica. With this stack the variation of the transmission curve is not modified by a variation of Ti:Ta2O5 thickness less than $3 \%$ which it is verified in our case.

The details of the thickness measurement based on spectrophotometric measurement and calculation of the mask has been explained in details in a previous paper [13].

Finally with a better sensitivity $(0.15 \%)$ on the thickness measurement we optimized the shape of the masks and we obtained uniformity of $0.3 \%$ in the central region $(\varnothing 160 \mathrm{~mm})$. In this configuration two real substrates ( $\varnothing 340 \mathrm{~mm}, 200 \mathrm{~mm}$ thick) were coated with a High Reflective stack $(2,8 \mu \mathrm{m})$ for the Input Test Mass of Advanced LIGO (HR ITM). The uniformity of the coating has been estimated by subtraction of the wavefront before and after coating (Fig 4). Even if the uniformity and sagitta were close to the requirements, the shape with a linear valley through the center and without cylindrical symmetry resulted in high Zernike coefficients (2.26 nm for Z40 for example) out of specifications.

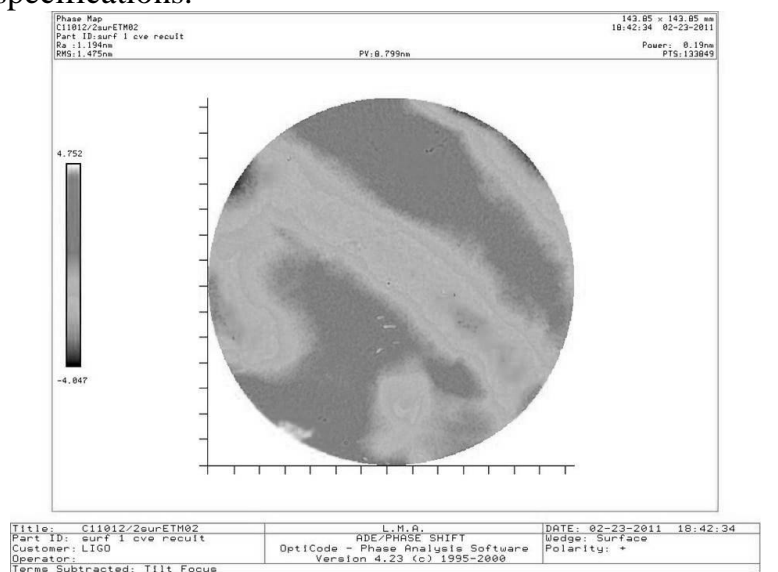

Fig 4: ETM 02- Coating uniformity- (subtraction of the mirror wavefront after and before coating) on $\varnothing 150 \mathrm{~mm}$

This result led us to test other configurations with revolution motion around the center of the substrate.

\section{The single rotation configuration:}

In this configuration the center of the substrate has been fixed concentrically with the axis of rotation of the substrate holder: the motion was just a single rotation around the center of the substrate. We have adjusted the position of the targets in order to optimize the uniformity. As for the twin mirrors, the coating thickness was measured with the spectrophotometer on 7 samples placed on the diameter. In this case due to the motion, we should find symmetric uniformity around the center of the substrate. But when the coating thickness uniformity becomes better than $0.25 \%$ we found dubious asymmetry which did not allow us to progress.

Finally we have used a large thin silica disk $(\varnothing 310$ $\mathrm{mm}, 6 \mathrm{~mm}$ thick) fixed on the fake metallic substrate. The thickness was measured along a diameter with a step of $10 \mathrm{~mm}$. With these plates, by optimization of the target positions, we have obtained more symmetrical uniformities. On the central $160 \mathrm{~mm}$ the uniformity was $0.2 \%$ for $\mathrm{Ti}: \mathrm{Ta}_{2} \mathrm{O}_{5}$ layers (Position c) and silica (Position B) (Fig. $6 \& 7$ ). 


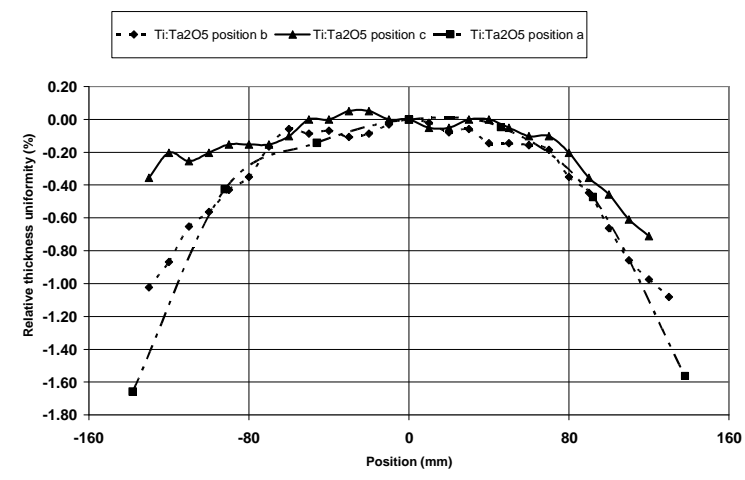

Fig 6- Ti:Ta2O5 Uniformity on $\varnothing 280 \mathrm{~mm}$

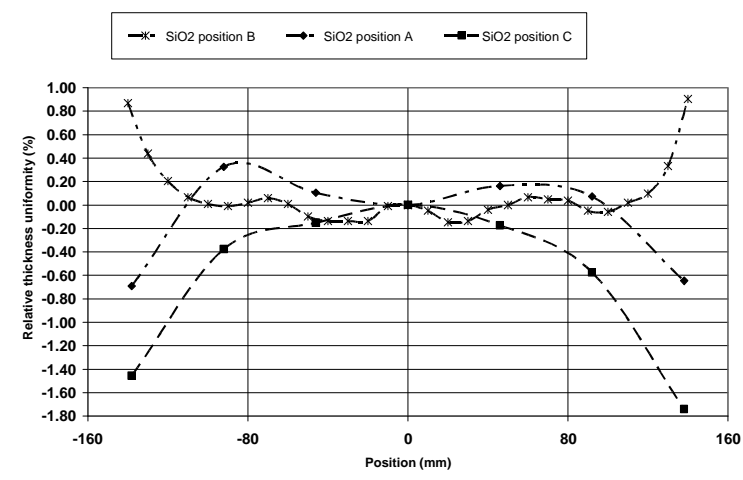

Fig 7- $\mathrm{SiO}_{2}$ Uniformity on $\varnothing 280 \mathrm{~mm}$

With the optimized positions we coated a HR ITM stack on one thin plate. We measured on one diameter every $10 \mathrm{~mm}$ the transmission spectrum. For each point we calculated the wavelength reference $\lambda c$ (middle of the Full Width Height Maximum-FWHM). The thickness uniformity was directly deduced from the variation of $\lambda c$ (Fig 8). On the central $160 \mathrm{~mm}$ we obtained around $0.15 \%$ of uniformity corresponding to about $4 \mathrm{~nm}$ PV.

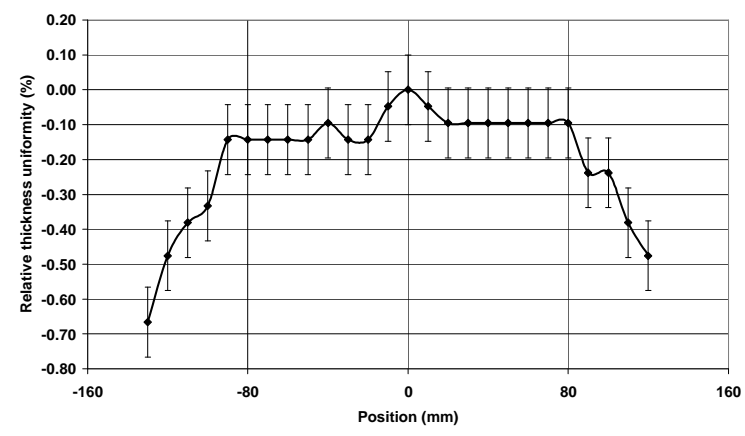

Fig 8- HR ITM Uniformity on $\varnothing 280 \mathrm{~mm}$
We decided to coat consecutively two real substrates $(\varnothing$ $340 \mathrm{~mm}, 200 \mathrm{~mm}$ thick) for Advanced LIGO. The results are quite similar for both mirrors: absorption $(0.3 \mathrm{ppm})$ and scattering $(5 \mathrm{ppm})$ on the central $\varnothing 160$ $\mathrm{mm}$ area respected the specifications. Transmissions are respectively 1.412 and $1.415 \%$ then the transmission matching (0.002) is better than the 0.01 requested. After coating and annealing, the measured flatness (Fig. 9) on the $160 \mathrm{~mm}$ central area is $0.3 \mathrm{~nm}$ rms (2.3 nm PV). The Zernike coefficients issued from the wavefront measurement were all in the specifications $(<0.5 \mathrm{~nm})$.

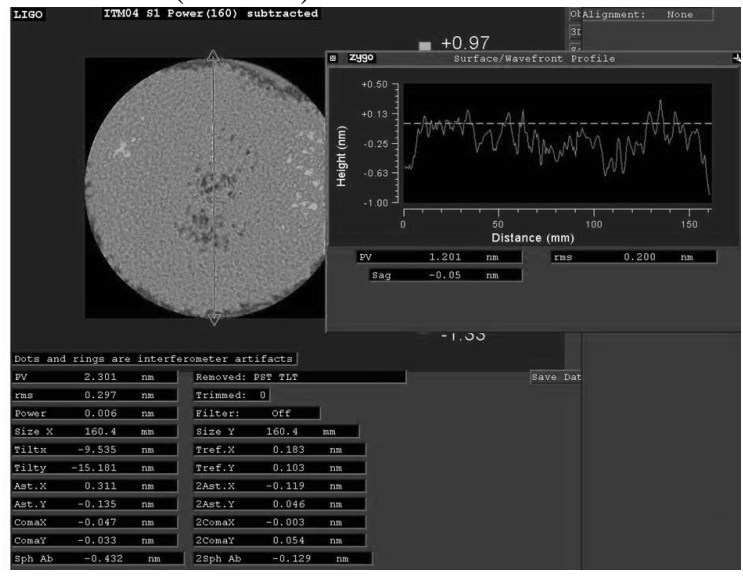

Fig 9 - ITM $\mathrm{N}^{\circ} 4$-Wavefront measurement on $\varnothing 160 \mathrm{~mm}$

We also coated two HR ETM stack on scale 1 substrate. If the absorption and scattering losses are on the same level than the ITM, the wavefront was different (fig. 10) due to the doubled thickness versus the ITM stack. The flatness was $0.76 \mathrm{~nm} \mathrm{rms}$ and the primary spherical aberration was out of specifications $(1.3 \mathrm{~nm})$.

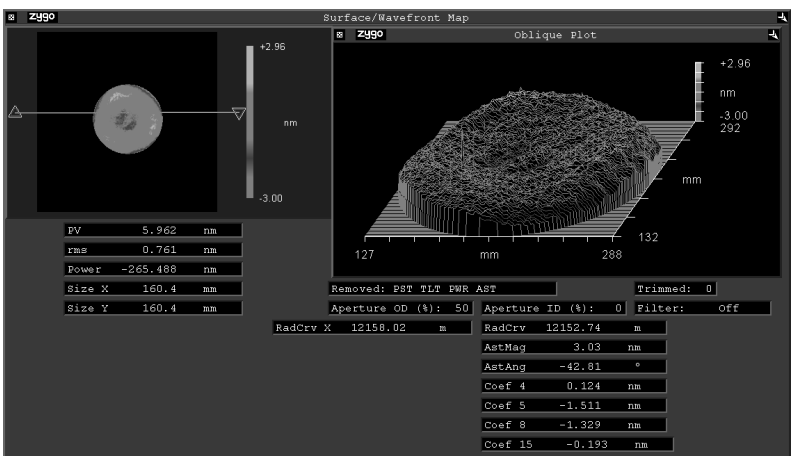

Fig 10- ETM N³- Wavefront measurement on $\varnothing 160$ $\mathrm{mm}$

Wavefront measurements have been used to simulate round trip losses in Fabry-Perot cavities. We obtained $60 \mathrm{ppm}$ for one couple ITM-ETM and $46 \mathrm{ppm}$ for the 
ETM alone. These values are slightly higher than the requirements.

With the single rotation we are able to coat the Input Test Mass in respect to the specifications for the Advanced Gravitational Wave detectors but the flatness is not enough for the End Test Mass with thicker coatings.

\section{The planetary system:}

Planetary system are regularly used in coating chambers for planarize [14] [15]. In our case it is necessary to coat two large heavy substrates $(\varnothing 350 \mathrm{~mm}, 200 \mathrm{~mm}$ thick, $40 \mathrm{~kg}$ ) in the same batch. We have planned to modify the twin mirrors configuration. The axis of rotation used for the twin mirrors gives the motion of the whole substrate holder (Ws) and for each substrate we added a second rotation (Wp) around their center with gears (Fig. 11). The ratio of the gears leads

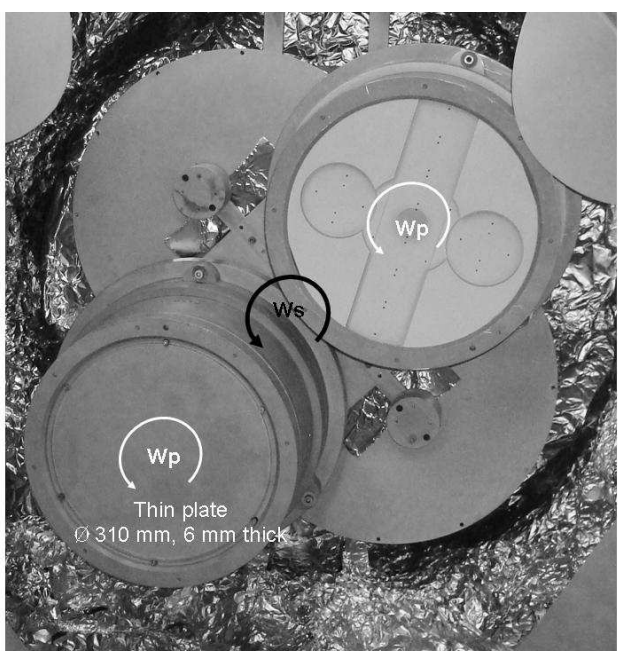

Fig 11-Planetary system with fake metallic substrate and thin plate

to a period of 930 seconds. With these parameters we could simulate the thickness achievable with this planetary motion coupled with the best masks used in the twin mirrors configuration. The simulations are based on the real plume profiles issued from deposition made on aluminium plates (Fig. 12). We used a classical method to extract the profile [14]. The analysis of the photograph of the interference patterns allowed us to reconstruct the plume profile with a model based on Gaussian functions with adjusted parameters. By calculating the cycloidal trajectory of each point of the substrate through the plume profile we were able to calculate the thickness deposited. Finally we were able to simulate the uniformity for the HR ITM and HR ETM stacks (Fig. 13 \& 14).

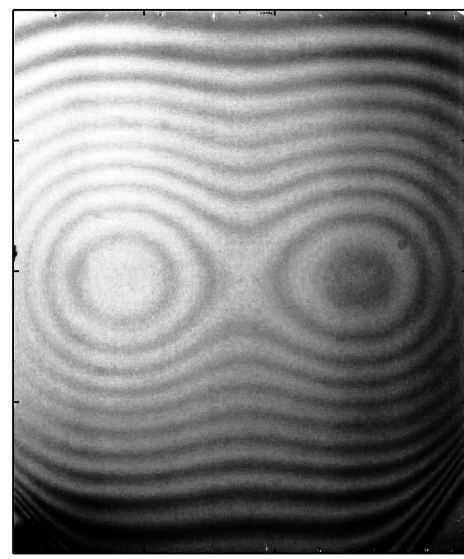

Fig. 12- Aluminium plate with a static deposition of silica
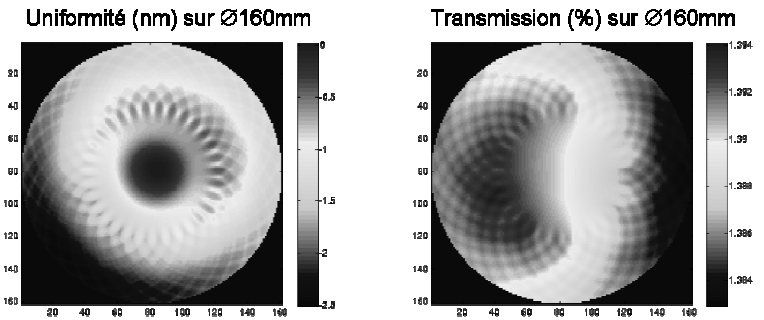

Fig. 13 - HR ITM- Left: Uniformity on $160 \mathrm{~mm}$ - Right: Transmission on $160 \mathrm{~mm}$

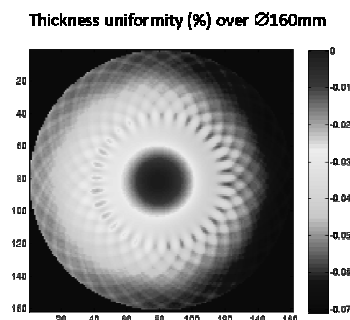

VY $\sim 4 \mathrm{~nm} \&$ RMS $\sim 0.85 \mathrm{~nm}$

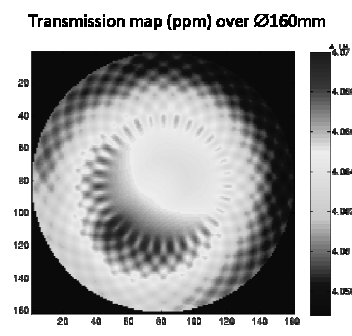

$T=4.06+/-0.0065 \mathrm{ppm}$
Fig. 14 - HR ETM -Left: Uniformity on 160 mm- Right:

Transmission on $160 \mathrm{~mm}$

For both stacks Sagitta and Zernike coefficients respected the requirements. The RTL simulated with these maps are less than the specified $50 \mathrm{ppm}$ (Table 1).

\begin{tabular}{|l|c|c|c|}
\hline & ITM & ETM & ITM+ETM \\
\hline Sagitta (nm) & 1.4 & 2.4 & \\
\hline RTL aVIRGO (ppm) & 8 & 16 & 15 \\
\hline RTL aLIGO (ppm) & 3 & 26 & 28 \\
\hline
\end{tabular}

Table 1- RTL simulated with planetary motion

The planetary system has been installed in our large coating chamber and first tests have been made with the masks used for the twin mirrors. As previously thicknesses were measured by spectrophotometric analysis on large thin plates. Thickness uniformity on Ti: $\mathrm{Ta}_{2} \mathrm{O}_{5}$ and silica were around $1 \%$ on $300 \mathrm{~mm}$ and 
$0.4 \%$ in the central $160 \mathrm{~mm}$. New masks were calculated and preliminary results are promising with uniformity around $0.15 \%$ in the central $160 \mathrm{~mm}$ and $0.4 \%$ on $300 \mathrm{~mm}$.

With these masks we have coated HR ITM and HR ETM stack. If all the parameters were acceptable for the ITM, some Zernike coefficients and RTL were too high for the ETM. New iterations for the mask are in progress.

\section{Conclusion:}

The second generation of gravitational waves detectors is under construction. One of the key points for the gain of one order of magnitude in sensitivity is the flatness of the large mirrors ( $\varnothing 350 \mathrm{~mm}, 200 \mathrm{~mm}$ thick, $40 \mathrm{~kg}$ ) forming Fabry-Perot cavities which are located in the arms of the Michelson interferometer. Sub-nanometer flatness is required on the central $160 \mathrm{~mm}$ area for both HR ITM and HR ETM coatings. The coating process shall not change the substrate sagitta by more than $8 \mathrm{~nm}$ and not add surface figure Zernike terms higher than second order with amplitude $>0.5 \mathrm{~nm}$. Three configurations have been studied for the motion of the substrate. If the twin mirrors configuration has allowed reaching good flatness the lack of symmetry around the center of the substrate is unacceptable to reach the specifications of the Zernike coefficients. With a single rotation motion and adjustments of the targets parameters we reached the requirements for the thinner HR ITM stack $(3 \mu \mathrm{m})$ and the first real ITM mirrors were delivered for Advanced LIGO. The thickness of the HR ETM stack $(6 \mu \mathrm{m})$ leads to too high Zernike coefficients. As the simulations of the planetary motion have given results in the specifications for all the parameters we installed this system in our large coating chamber. First experimental results are promising and we have already reached the requirements for the ITM mirrors. New iterations are needed for the thicker HR ETM stack.

Acknowledgment: The results described in this paper were possible thanks to the work of several staff members at the Laboratoire des Matériaux Avancés. In particular we would like to thank Mrs Pignard.

Virgo has been constructed and is operated by the Centre National Recherche Scientifique and the InstitutoNazionale di Fisica Nucleare. LIGO was constructed by the California Institute of Technology and Massachusetts Institute of Technology with funding from the National Science Foundation and operates under cooperative agreement PHY-0757058. This paper has LIGO Document Number LIGO-P1200174-v1.

\section{REFERENCES}

[1] David T. Wei- "Ion beam interference coating for ultralow optical loss"- APPLIED OPTICS/ Vol. 28, No. 1415 /July 1989

[2] The VIRGO Collaboration et al.-"The VIRGO large mirrors: a challenge for low loss coatings"- Class Quantum Grav. 21 S935

[3] T. Accadia et al. for the Virgo collaboration "Virgo: a laser interferometer to detect gravitational waves"-Journal of Instrumentation 7, P03012 doi:10.1088/1748-0221/7/03/P03012

[4] F Beauville, D Buskulic, R Flaminio, F Marion, A Masserot, L Massonnet, B Mours, F Moreau, J Ramonet, E Tournefier, D Verkindt, O Veziant, M Yvert, R Barillé, V Dattilo, D Enard, F Frasconi, A Gennai, P La Penna, M Loupias, F Paoletti, L Bracci, G Calamai, E Campagna, G Conforto, E Cuoco, I Fiori, G Guidi, G Losurdo, F Martelli, M Mazzoni, B Perniola, R Stanga, F Vetrano, A Viceré, D Babusci, G Giordano, J-M Mackowski, N Morgado, L Pinard, A Remillieux, F Acernese, F Barone, E Calloni, R De Rosa, L Di Fiore, A Eleuteri, L Milano, K Qipiani, I Ricciardi, G Russo, S Solimeno, M Varvella, F Bondu, A Brillet, E Chassande-Mottin, F Cleva, T Cokelaer, J-P Coulon, B Dujardin, J-D Fournier, H Heitmann, C N Man, F Mornet, J Pacheco, A Pai, H Trinquet, J-Y Vinet, N Arnaud, M Barsuglia, M A Bizouard, V Brisson, F Cavalier, M Davier, P Hello, P Heusse, S Kreckelberg, C Boccara, V Loriette, J Moreau, V Reita, P Amico, L Bosi, L Gammaitoni, M Punturo, F Travasso, H Vocca, L Barsotti, S Braccini, C Bradaschia, G Cella, C Corda, A Di Virgilio, I Ferrante, F Fidecaro, A Giazotto, E Majorana, L Holloway, R Passaquieti, D Passuello, R Poggiani, A Toncelli, M Tonelli, L Brocco, S Frasca, C Palomba, P Puppo, P Rapagnani and F Ricci - "The Virgo large mirrors: a challenge for low loss coatings"Classical and Quantum Gravity 21 n $^{\circ 5}$ (2004) S935S945

[5] T. Accadia et al for the Virgo collaboration "Status of the Virgo project"-Classical and Quantum Gravity 28 (2011) 025005 (25pp)

[6] T. Accadia et al. (The Virgo Collaboration), "Advanced Virgo Technical Design Report," Virgo technical note VIR-0128A-12 (2012), https://tds.egogw.it/itf/tds/.

[7] G. M. Harry (for the LIGO Scientific Collaboration), "Advanced LIGO: the next generation 
of gravitational wave detectors," Classical and Quantum Gravity 27, 084006 (2010).

[8] Gregory M Harry, Andri M Gretarsson, Peter R Saulson, Scott E Kittelberger, Steven D Penn, William J Startin, Sheila Rowan, Martin M Fejer, D R M Crooks, Gianpietro Cagnoli, Jim Hough and Norio Nakagawa "Thermal noise in interferometric gravitational wave detectors due to dielectric optical coatings"- 2002 Class. Quantum Grav. 19

[9] G. Harry, T.P. Bodiya, R.DeSalvo "Optical Coatings and Thermal Noise in Precision Measurement" Edited by Greg Harry

[10] L. Pinard, B. Sassolas, R. Flaminio, D. Forest, A. Lacoudre, C. Michel, J.L. Montorio, N. Morgado"Towards a new generation of low-loss mirrors for the advanced gravitational waves interferometer"-Optics Letters, vol. 36 n $^{\circ} 8$ (2011) 1407

[11] TFCalc by Software Spectra, Inc. http://www.sspectra.com/

[12] Optical Constant: Matlab code developped at LMA

[13] B. Sassolas, Q. Benoit, R. Flaminio, D. Forest, J. Franc, M. Galimberti, A. Lacoudre, C. Michel, J.L. Montorio, N. Morgado, L. Pinard- "Twin mirrors for the laser interferometric gravitational-wave detectors" Applied Optics, Vol. 50 n 13 (2011) 1894

[14] SPECTOR Large Area Planetary Ion Beam Deposition System. VEECO Instruments Inc. http://www.veeco.com/

[15] M. Gross, S. Dligatch and A. Chtanov"Optimization of coating uniformity in an ion beam sputtering machine using a modified planetary motion method"-Applied Optics, vol. 50, March 2011 